Musées, Patrimoine et Culture scientifiques et techniques

$182 \mid 2019$

mars-avril 2019

\title{
Le nouveau Muséum de Bordeaux : convivialité et simplicité
}

Nathalie Mémoire

\section{OpenEdition}

\section{Journals}

Édition électronique

URL : http://journals.openedition.org/ocim/2359

DOI : 10.4000/ocim.2359

ISSN : 2108-646X

Éditeur

OCIM

Édition imprimée

Date de publication : 1 mars 2019

Pagination : $50-53$

ISSN : 0994-1908

Référence électronique

Nathalie Mémoire, "Le nouveau Muséum de Bordeaux : convivialité et simplicité », La Lettre de l'OCIM [En ligne], 182 | 2019, mis en ligne le 01 mars 2020, consulté le 16 mars 2020. URL : http:// journals.openedition.org/ocim/2359; DOI : https://doi.org/10.4000/ocim.2359

Ce document a été généré automatiquement le 16 mars 2020.

Tous droits réservés 


\title{
Le nouveau Muséum de Bordeaux : convivialité et simplicité
}

\author{
Nathalie Mémoire
}

Accueil du Muséum de Bordeaux

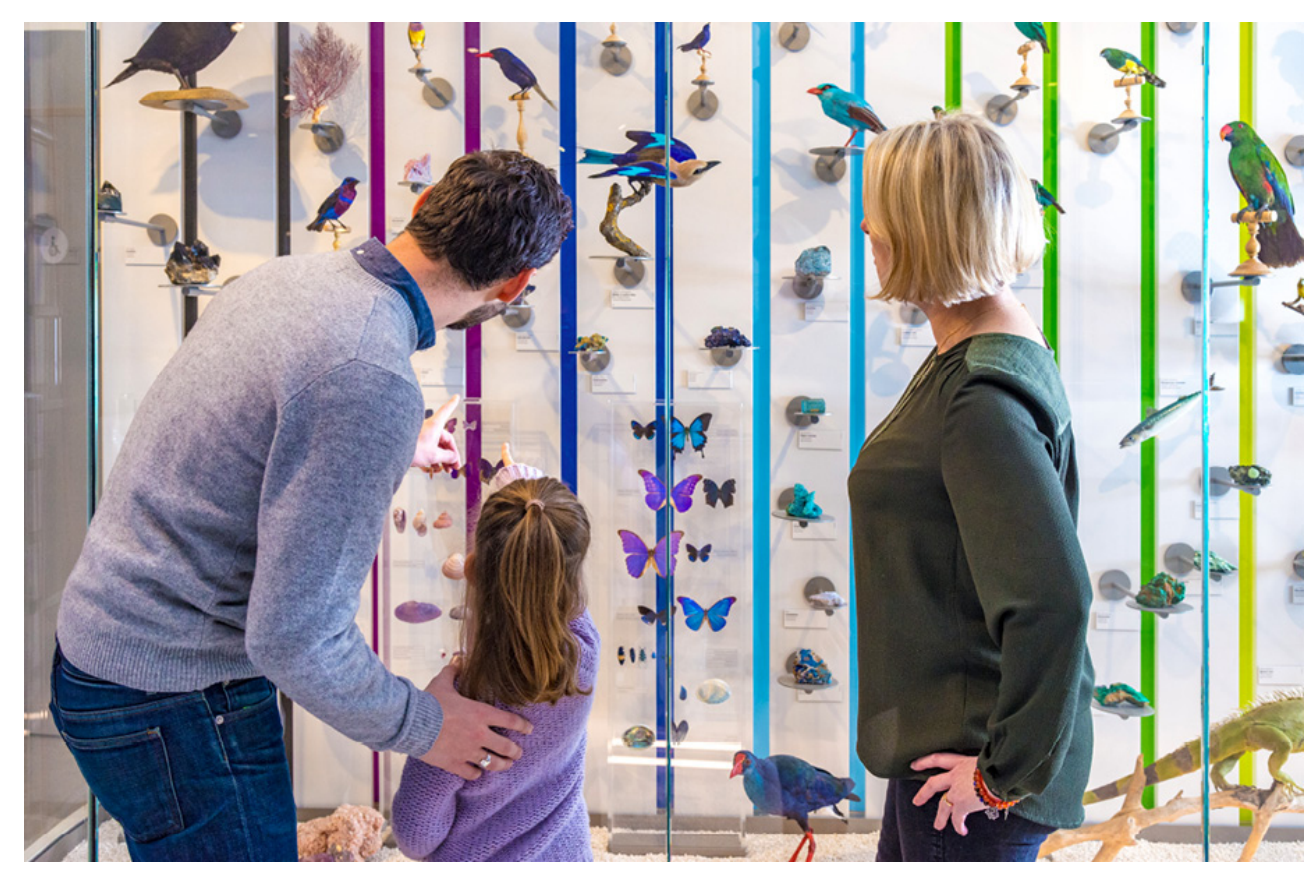

(c) F. Deval

Après 10 ans de fermeture, le Muséum de Bordeaux a ouvert ses portes au public le 31 mars dernier. Que s'est-il passé durant cette période?

En septembre 2000, un premier projet de rénovation-

extension est remis à la Ville. L'objectif est de mettre en conformité le bâtiment et d'améliorer l'accueil des publics, d'optimiser les conditions de conservation des collections, et de proposer des pistes pour une extension. Plusieurs propositions sont étudiées : déménager dans un nouvel édifice à construire en périphérie, agrandir 
l'existant en réaménageant l'un ou l'autre des bâtiments voisins au sein du Jardin public. L'une des propositions d'extension dans le jardin est privilégiée. De 2003 à 2005, des études de diagnostic, de faisabilité et de programmation sont menées par le cabinet d'architecture A2M.

En juillet 2006, l'enveloppe budgétaire fixée, la décision est votée par le Conseil municipal. L'extension se fera dans les locaux qui seront libérés en 2007 par le Jardin botanique transféré sur la rive droite de la Garonne. L'équipe du Muséum et le programmiste ont 4 mois pour récrire le projet adapté à la nouvelle configuration. Ce document précise les contenus scientifiques, actions pédagogiques, mise en exposition et gestion des collections. Il propose un parcours muséographique actualisé et innovant, plaçant les problématiques environnementales au cœur du projet. Ce projet scientifique et muséographique constitue le PSC de l'établissement. Le programme architectural est précisé en parallèle.

Le concours d'architecture est lancé en octobre 2006. En décembre, 4 équipes de maîtrise d'œuvre sont admises à concourir sur esquisse, choisies parmi 67 candidats. En mai 2007, le projet du groupement Basalt Architecture (mandataire) avec Die Werft Muséographie (scénographie), Coplan-Otéis (bureau d'étude) et Impédance (acoustique) est retenu. Il se distingue par :

- le traitement de la galerie XIX ${ }^{\mathrm{e}}$ siècle dans le respect de l'architecture d'origine ;

- la création d'un vaste espace d'exposition temporaire en sous-sol ;

- la création d'une transition harmonieuse entre le parc et les espaces intérieurs du Muséum grâce à une entrée en sous-sol accessible via une déambulation en pente douce ;

- la mise en valeur des collections dans une scénographie contemporaine et épurée ;

- l'intégration des dispositifs multimédia attendus dans les parcours d'exposition.

Les différentes composantes du projet concours sont affinées dans l'Avant-Projet Sommaire (APS). Des études techniques portant sur le sous-sol et les bâtiments sont réalisées, ainsi qu'une analyse des attentes du Muséum dont les équipes précisent les synopsis des parcours d'exposition et la sélection des spécimens. Des plans des espaces et une estimation détaillée des coûts sont fournis par la maîtrise d'œuvre.

Ces éléments sont approfondis dans l'Avant-Projet Définitif (APD), voté au Conseil municipal de juillet 2010, et enfin dans le projet proprement dit (PRO). Ils sont ensuite détaillés dans le Document de Consultation des Entreprises (DCE) lors des appels d'offres.

Le projet comporte trois bâtiments, les avancées des uns conditionnant celles des autres. L'Hôtel de Lisleferme, qui regroupait tous les services du Muséum, sera entièrement dédié à l'accueil du public et à la diffusion de la culture scientifique (expositions, animations, conférences). La bibliothèque, les bureaux et les ateliers techniques sont transférés dans le bâtiment en pierre des anciennes serres du Jardin botanique, réhabilité en "pavillon administratif» en 2011. Inauguré en 2012, le Centre de Conservation des Collections a été construit pour répondre aux exigences de conservation préventive et assurer toutes les fonctions liées à la gestion des collections. Les collections y sont déplacées ; une partie seulement reviendra dans le nouveau parcours, par roulement, à la faveur des expositions. 
En 2012, deux appels d'offres infructueux auprès des entreprises du bâtiment imposent une révision du projet architectural. La réorganisation des espaces et l'agrandissement sont réétudiés afin de limiter certaines contraintes techniques. L'entrée du musée, initialement prévue en sous-sol est finalement ramenée au rezde-chaussée, tout comme l'espace d'accueil attenant. L'extension en sous-sol de 500 $\mathrm{m}^{2}$ est entièrement construite sous le jardin, et non plus en sous-œuvre sous le bâtiment.

Pour finir, vous avez joué de malchance.

En effet, le samedi 26 mai 2018, un violent orage de grêle compromet la poursuite de l'installation des collections. Les chéneaux de la toiture débordent dans les combles, provoquant un dégât des eaux majeur. Tous les espaces sont touchés, excepté le soussol. Le lundi, l'équipe s'affaire pour retirer les collections déjà installées ; 75 spécimens devront être restaurés. Quelques semaines sont nécessaires pour évaluer l'ampleur des dégâts sur le bâtiment, plafonds et parquets, l'installation électrique et le mobilier muséographique, et trois mois pour réaliser les travaux. Initialement prévue en novembre 2018, la réouverture du Muséum doit être repoussée à 2019.

Entre temps il a fallu relire les derniers textes, tester les multimédias, mais aussi effectuer les derniers recrutements, passer la nouvelle organisation du personnel en comité technique, finaliser et réaliser les recettes du site internet, valider les levées de réserves sur le bâtiment et les équipements, organiser la commission de sécurité, accueillir la presse et les mécènes...

Quelles sont les sources d'inspiration qui vous ont guidées pour imaginer le nouveau muséum?

Les inspirations ont été multiples, les visites d'autres établissements ainsi que les échanges avec les collègues ont été déterminants tant pour l'écriture du " musée " rêvé que pour la concrétisation finale du projet. L'observation de nombreux musées et expositions, tant à l'étranger qu'en France, m'ont permis d'alimenter notre réflexion : ce qui marche ou ne marche pas, ou plus, ce qui est passé de mode très vite et ce qui reste une valeur sûre au-delà des décennies et des nouveaux publics, ce qui peut être actualisé et revisité à partir d'une base intemporelle.

Un hiver passé au Danemark, grâce à une bourse du gouvernement danois pour l'étude de la muséologie au Danemark, reste à l'origine de mon "musée rêvé ». Loin des « temples de la culture » dont il fallait mériter l'accès encore souvent en France, les conservateurs danois mettaient depuis longtemps en avant la convivialité et la simplicité.

Ainsi, l'idée du Carré détente à mi-parcours, avec la vue sur le jardin et des revues à disposition, me vient du musée d'Art moderne de Louisiana, à Humlebaek et la conviction du conservateur de l'époque, Hans Erik Wallin, que les visiteurs peuvent venir juste pour passer un bon moment et ainsi se familiariser avec les collections, même et surtout s'ils n'y connaissent rien.

Les voyages de l'Ocim à l'étranger, en compléments de voyages personnels, ont été pour moi des sources d'inspiration essentielles dans la conduite du projet. Voici, en liste à la Prévert, quelques musées, sites ou expositions qui ont pu nous inspirer, certains anciens mais toujours séduisants, d'autres plus récents : 
- le Musée des Beaux-Arts de Toronto (avec l'Ocim, 1997) pour les médiateurs ambulants et leur chariot de collections destiné à susciter chez le public l'envie d'échanger ;

- l'Historial de la Vendée (ouvert en 2006, visité en 2009) pour les sièges pliants à disposition dans les salles et les contenus audio scénarisés; pour les étiquettes avec photographie des spécimens et non des renvois à des listes de numéros ;

- le Museon de la Haye (avec l'Ocim, 1989) pour la modularité des espaces (cloisons mobiles) et les vitrages discrets pour la mise à distance ;

- les expositions itinérantes déclinées pour les moins de 6 ans de l'association belge Apex qui nous a donné l'idée, en 2006, de créer l'exposition Tout les bébés, préfiguration de l'exposition d'ouverture de ce nouvel espace, le Musée des Tout-petits ;

- l'espace Gens de Garonne, à Couthure (Lot-et-Garonne), en 2006, pour le show multimédia ;

- l'exposition Bêtes et Hommes de la Cité des Sciences et de l'Industrie, en 2010, pour la classification imaginaire de Jorg Luis Borgès ;

- le Musée de la science CosmoCaixa à Barcelone, en 2016, pour la thématique des formes géométriques dans la nature ;

- le vol de la Chouette effraie au Muséum d'Orléans, à la fin des années 1980, pour le développement du vol du Martin-pêcheur, décomposé grâce une série de montages taxidermiques.

\section{Quelles convictions vous ont animé ?}

Un musée, ce sont des collections, mais ce sont aussi, et peut-être d'abord, des hommes et des femmes : ceux qui ont réuni ces collections, ceux qui les entretiennent, ceux qui les étudient, ceux qui les font vivre et parler, ceux qui viennent les voir, les questionner ou les admirer.

Un musée doit être un lieu de plaisir et d'émerveillement où sont sollicités la vue mais aussi l'ouïe et lorsque c'est possible le toucher. C'est seulement si cette première approche est réussie que les autres objectifs ont une chance de séduire le public mis ainsi en condition de réception. C'est aussi un lieu d'éducation informelle ou plutôt non formelle comme l'énonce Daniel Jacobi, mais pas d'injonction, un lieu qui doit permettre le questionnement, un lieu qui montre la construction de connaissances provisoires et non l'affirmation dogmatique de savoirs, un lieu de transmission et d'échanges intergénérationnels.

Le musée a un rôle social et les collections d'un muséum permettent de s'adresser à tous, en portant des récits très variés, pouvant d'adapter à tous les âges et à tous les niveaux d'instruction dès lors que l'information est mise à disposition si on veut la chercher pour en voir ou en savoir plus.

Nous nous sommes efforcés de traduire ces convictions dans les récits portés par les collections, par le cahier des charges de la scénographie auquel a répondu la maîtrise d'œuvre et les entreprises, tant dans la réorganisation du bâtiment (cependant très contraint par le patrimoine historique bâti) que par les couleurs, lumières et volumes, et les différents dispositifs scénographiques mis en place, enfin par les 
choix réalisés pour la médiation, alliant numérique et humain. Mais au final, seul le public validera ou non la pertinence de ces choix.

Racontez-nous la visite au nouveau muséum : quelle en est l'originalité ? Quels en sont les inattendus?

Le nouveau parcours muséographique se déploie autour de la question de la place de l'Homme dans la nature. Les collections, patrimoine naturel, sont désormais présentées pour soutenir des propos scientifiques et sociétaux qui interrogent les citoyens. Un effort considérable a été porté sur la médiation avec la volonté d'offrir à tous les publics différents niveaux d'exploration, de découverte et d'émerveillement. Un spectacle multimédia offre à chacun une expérience immersive. Le visiteur pourra compléter sa visite à l'aide des bornes multimédias ou en échangeant avec les médiateurs.

Le Muséum de Bordeaux enrichit son parcours permanent en diversifiant ses invitations avec des expositions temporaires et des expositions semi-permanentes.

Attaché à l'éveil des très jeunes publics, le Muséum se distingue également en créant Le Musée des Tout-petits, réservé aux enfants de moins six ans.

La rénovation-extension a permis de conjuguer différents objectifs comme la mise aux normes du bâtiment, le confort des publics, la mise en œuvre des modalités de conservation préventive et le respect de l'environnement par différents procédés de réduction de consommation d'énergie. Elle a également donné un nouveau souffle à ce lieu tout en mettant en valeur les éléments significatifs de l'édifice.

Pas de muséum sans équipe, comment s'organisent les compétences professionnelles dans votre établissement?

L'équipe se compose aujourd'hui de 21 permanents et un nombre variable de vacataires :

- une équipe conservation-production comptant 8 permanents. Le travail de l'équipe de conservation est le socle sans lequel il n'y aurait pas de musée. Ils assurent la conservation préventive, la régie des collections, l'inventaire et l'étude. Elle est enrichie par des vacataires, spécialisés dans les différentes catégories de collections, mais dont le renouvellement est toujours soumis à négociation ; le soutien financier du ministère de l'Enseignement supérieur, de la Recherche et de l'Innovation a été déterminant pour leur recrutement tout au long du projet. L'équipe technique, qui assure la production et la maintenance, vient en appui des prestataires, et crée de manière autonome des dispositifs d'exposition temporaire et de médiation.

- une équipe diffusion au public composée de 5 permanents, de personnel occasionnel et de l'appui de 2 enseignants mis à disposition. La mission de conservation n'a de sens que si elle est couplée avec celle de diffusion au public. Pour le nouveau parcours, l'équipe exposition a été en charge de la préparation de l'écriture des textes sur tous les supports et du suivi de leur exécution par les prestataires, et travaille sur les expositions temporaires à venir. À l'ouverture, l'équipe de médiation compte 10 médiateurs scientifiques, vacataires à mi-temps, qui assureront une médiation ambulante active.

- une équipe administrative (dont billetterie-boutique) composée de 5 permanents et deux vacataires. Elle permet aux missions fondamentales du musée de se réaliser, de 
faire face à la complexité administrative, et de contribuer directement à l'accueil du public.

- une équipe communication-mécénat composée de 2 permanents, de vacataires et de stagiaires. Ce service fait face aux nouvelles exigences de la réouverture, entre recherche de partenaires financiers, création du site internet, animation des réseaux sociaux et relations presse. C'est celui qui devra le plus vite être renforcé.

- enfin des tâches sont externalisées comme la sécurité et le nettoyage des locaux, assuré majoritairement par la mutualisation des agents d'entretien des écoles. La taxidermie et la restauration des collections sont confiées à des prestataires.

Quels conseils donneriez-vous à vos collègues qui auront demain à mener ce travail de rénovation?

Du temps

Visiter d'autres musées

Bien connaître ses collections, leur potentiel, leurs faiblesses

Anticiper largement les programmes de restauration et d'acquisition

Se battre pour obtenir du temps pour le déménagement et la réinstallation

Faire des maquettes. Ne pas hésiter à les corriger

Tester les dispositifs, faire des préfigurations et des prototypes avec des panels de visiteurs

Ne pas se décourager

S'entourer de jeunes pour la dynamique et les nouvelles façons de travailler

Écouter les anciens qui ont l'expérience et qui ont déjà essayé des tas de choses, qui marchent ou pas

Ne pas céder à la mode

Savoir ce qu'on veut dire et montrer, et le défendre

\section{RÉSUMÉS}

$\mathrm{Au}$ moment où le Muséum de Bordeaux prend un nouveau départ, Nathalie Mémoire, sa conservatrice, interrogée par l'équipe de l'Ocim, revient sur les différentes étapes qui ont jalonné sa rénovation-extension et sur les réflexions qui ont alimenté l'élaboration de ce projet de «musée rêvé ».

INDEX

Mots-clés : Rénovation, muséum, Bordeaux 


\section{AUTEUR}

\section{NATHALIE MÉMOIRE}

Nathalie Mémoire est conservatrice et directrice du Muséum de Bordeaux.

n.memoire@mairie-bordeaux.fr 